\title{
Solving the Multi Observer 3D Visual Area Coverage Scheduling Problem by Decomposition
}

\author{
Helman I. Stern ${ }^{1}$, Moshe Zofi ${ }^{1,2}$, Moshe Kaspi ${ }^{1}$ \\ ${ }^{1}$ Department of Industrial Engineering and Management, \\ Ben Gurion University of the Negev, Beer-Sheva, Israel \\ ${ }^{2}$ Department of Industrial Management, Sapir College, Sderot, Israel \\ E-mail: \{helman, zofi, moshe\}@bgu.ac.il \\ Received April 26, 2011; revised May 15, 2011; accepted June 11, 2011
}

\begin{abstract}
This paper presents two solution methodologies for the Visual Area Coverage Scheduling problem. The objective is to schedule a number of dynamic observers over a given 3D terrain such that the total visual area covered (viewed) over a planning horizon is maximal. This problem is a more complicated extension of the Set Covering Problem, known to be Np-Hard. We present two decomposition based heuristic methods each containing three stages. The first methodology finds a set of area covering points, and then partitions them into routes (cover first, partition second). The second methodology partitions the area into a region for each observer, and then finds the best covering points and routes (partition first, cover second). In each, a last stage determines dwell (view) times so as to maximize the visible coverage smoothly over the terrain. Comparative tests were made for the two methods on real terrains for several scenarios. When comparing the best solutions of both methods the CF-PS method was slightly better. However, because of the increased computation time we suggest that the PF-CS method with a fine terrain approximation be used. This method is faster as partitioning the terrain into separate regions for each observer results in smaller coverage and routing problems. A sensitivity analysis of the number of observation points to the total number of terrain points covered depicted the classical notion of decreasing returns to scale, increasing in a convex manner as the number of observation points was increased. The best method achieved 100 percent coverage of the terrain by using only 2.7 percent of its points as observation points. Experts stated that the computer based solutions can save precious time and help plan observation missions with satisfying results.
\end{abstract}

Keywords: Heuristic Search, Genetic Algorithm, Computer Vision, Multi Agents, Meta-Heuristic

\section{Introduction}

The development of Geographical Information Systems (GIS) in recent years has made a large contribution to the ability to solve various terrain related problems efficiently. Problems such as; 1) finding the optimal path between two points over a rough terrain, and 2) locating observers in order to visually cover an area can now be solved using mathematical tools. Locating observers over a given area can be modeled as the Set Covering Problem (SCP). The traditional art gallery problem first proposed by Chvatal deals with the problem of finding the minimal number of observers required for complete visual coverage of a 2D polygon [1]. This problem is NP-hard, with popular approximations running in $O(n \log n)$ [2].
The approach does not model dynamic observers, the quality of coverage, nor does it consider costs.

The Visual Area Coverage Scheduling (VACS) problem offered here combines the two problems of; locating observation points over a $3 \mathrm{D}$ terrain, and routing multiple observers through them. Creating the best synchronization between the observers so as to view the maximal area of the terrain is the main objective. A second objective is to smooth coverage over the terrain. The VACS problem has been formulated and solved using a Genetic Algorithm in [3,4]. This paper introduces two decomposition based methodologies; 1) cover first, partition second (CF-PS) and 2) partition first, cover second (PF-CS), each containing three stages. The analysis is divided into finding observation points, routing observers through 
them and determining dwell times at each point. Placing static observers over a given $3 \mathrm{D}$ terrain was studied before using two heuristic approaches for graph approximations of the terrain. One is based on a rectangular grid representation [5], and the other on a Triangle Irregular Network (TIN) [6]. As far as we know, no previous work has been made on placing dynamic observers over a 3D terrain. Since many observation problems include a rapid change of locations, the VACS problem has an important practical use in surveillance operations.

The paper is organized as follows. Section 2 defines and formulates the VACS problem. Sections 3 and 4 present the two decomposition methodologies with illustrative examples of each. In section 5 a comparison of the two methodologies is made using real life $3 \mathrm{D}$ terrains. Section 5 also provides a discussion of the results, followed by conclusions.

\section{The VACS Problem}

\subsection{Problem Definition}

The VACS problem objective is to schedule a fixed number of observers $(Q)$, traversing a $3 \mathrm{D}$ terrain, over a fixed planning horizon $(T)$ such that; the total area covered is maximal and viewed uniformly subject to the following conditions: 1) there is a maximum time limit $(S)$ for remaining at a specific observation point, 2) every point on the terrain must be covered at least a certain amount of time $(Y \%$ out of $T), 3)$ no area is visible when an observer is on the move, 4) each move must exceed a minimal stated distance $(d), 5)$ movement from one point to another must be within the observer's limitations ( $\max$ slope angles, time, etc.), and 6) the observer has 360 degree vision.

\subsection{Problem Complexity}

Consider the case in which the observation dwell time $S$ is not limited (i.e. $S \rightarrow \infty$ ), the minimal distance between every two consecutive points $(d)$ is extremely large (i.e. $d \rightarrow \infty$ ) and the time required for movement between every pair of points in the terrain is very small (i.e. $t_{i, j} \rightarrow 0 \forall i, j$ ).

In this special case, no observer can have more than a single observation point along its route, and since there are no movement time limitations, each observer can be located at any required point in no time. The optimal solution to the VACS problem in this specific case is to find the $Q$ best observation points in which to place the $Q$ observers along the entire planning horizon. This is one of the forms of the Set Covering Problem (SCP) [7] which is a well known NP-Hard problem [8]. Since the
VACS problem can be reduced to SCP, we say that the VACS problem is also NP-Hard.

\subsection{Performance Measure}

Visibility and dead zones can be calculated both for $2 \mathrm{D}$ and $3 \mathrm{D}$ environments as shown in $[9,10]$. The total visible area observed in the terrain, by all observers at a given time $t$, is accumulated along the entire planning period. This accumulated number will be the total visibility performance measure. Maximizing the total area observed over the time horizon does not guarantee that every point will be seen by the observers. It is thus, necessary to consider the distribution of visible occurrences as well. This leads to a bi-criteria objective; 1) maximizing the number of visible points over the time horizon, and 2) trying to view each point an equal amount of time or at least a fixed percentage of time. It should be noted that both objectives may not be achieved simultaneously.

\subsection{Notation}

Let $P=\{1,2, \cdots, N\}$ be the set of all grid points describing the terrain. Time is broken into small, discrete segments $t=[0,1, \cdots, T]$, where $T$ represents the planning horizon. Let $S$ denote the maximal dwell time, such that no observer is allowed to stay more than $S$ units of time in one place. Let $d$ denote the minimum allowed distance between two consecutive observation points. Let $Y$ denote the percentage of time out of $T$ in which every point must be covered. Let there be $Q$ observers indexed as $(q=1,2, \cdots, Q)$. Let $P_{q, t}$ represent the location of observer $q$ at time $t$ where, $P_{q, t}=1,2, \cdots, N$. Let $A_{q, t}$ be a binary variable set to 1 , if observer $q$ is observing at time $t$, and 0 otherwise. The values of $N, T, S, Y, Q$ and $d$ are given constants while $P_{q, t}$ and $A_{q, t}$ are integer variables. Let $D$ be a $N \times N$ matrix whose common element $d_{i, j}$ represents the 3D distance from point $i$ to point $j$. Let $M T$ be a $N \times N$ matrix whose common element $t_{i, j}$ represents the time required to move from point $i$ to point $j$. Let $V$ be the 3D visibility matrix, whose common element $v_{i, j}$ equals 1 if point $j$ is visible from point $i$, and 0 otherwise. $V$ and $M T$ can be determined by the algorithms in $[10,11]$.

\subsection{Mathematical Formulation}

Problem 1: VACS Non-Linear Program

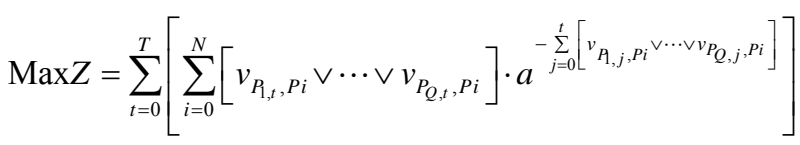

s.t. 


$$
\begin{gathered}
\prod_{t}^{t+S+1} A_{q, t}=0 ; \forall q, \forall t \\
d_{P_{q, t}, P_{q, m}}-d \cdot A_{q, t} \cdot A_{q, m} \cdot \prod_{t<k<m}\left(1-A_{q, k}\right) \geq 0 ; \forall q, \\
\forall t=0, \cdots, m-1, \forall m=0, \cdots, T \\
t_{\left(P_{q, t}, P_{q, t+1}\right)} \leq 1 ; \forall q, \forall t \\
\sum_{t=0}^{T}\left[v_{P_{1, t}, P i} \vee v_{P_{2, t}, P i} \vee \cdots \vee v_{P_{Q, t}, P i}\right]-Y \cdot T \geq 0 ; \forall i
\end{gathered}
$$

where $A_{q, t}=0,1, P_{q, t}=1, \cdots, N, q=1, \cdots, Q, t=0, \cdots, T$, $a>1$.

The objective function $Z$ in (1) represents the penalized total visibility throughout the entire planning horizon. The OR logic function returns the value of 1 , if at least one of the $Q$ observers sees point $i$ at time $t$. This term is multiplied by an exponential function which decreases according to the number of previous times the point was viewed. This smoothes out the visibility over all the points by insuring the more times a point has been viewed up to time $t$, the less value there is to view it again. Constraint (2) prevents an observer from staying in an observation point more than $S$ continuous segments of time. Constraint (3) insures a distance of at least $d$ when a move occurs. Constraint (4) is added to avoid non feasible solutions in which the observer moves to a point that is not adjacent to its current location in the first step of its path to the next observation point. Constraint (5) ensures that every point will be seen $Y$ percent of the time. A more detailed explanation for this formulation can be found in [3]. This formulation is untenable using mathematical programming techniques as it contains logical and nonlinear expressions in both its objective function and constraints. Hence, two heuristic solution approaches are proffered in the next two sections

\section{The Cover First, Partition Second (CF-PS) Heuristic}

\subsection{Overview}

This methodology has three phases. The first phase selects $M$ specific locations in the terrain to be visited by the observers. The number of observation points is fixed at $M=Q \cdot K$, where each observer will stop at $K$ observation points. Four alternative covering heuristics are developed for this purpose, which are described in the next section. The second phase solves a modified Vehicle Routing Problem (VRP) [12] heuristic to iteratively combine points until there are $Q$ routes, one for each observer. The observation time at each point along every route is determined using linear programming in the third

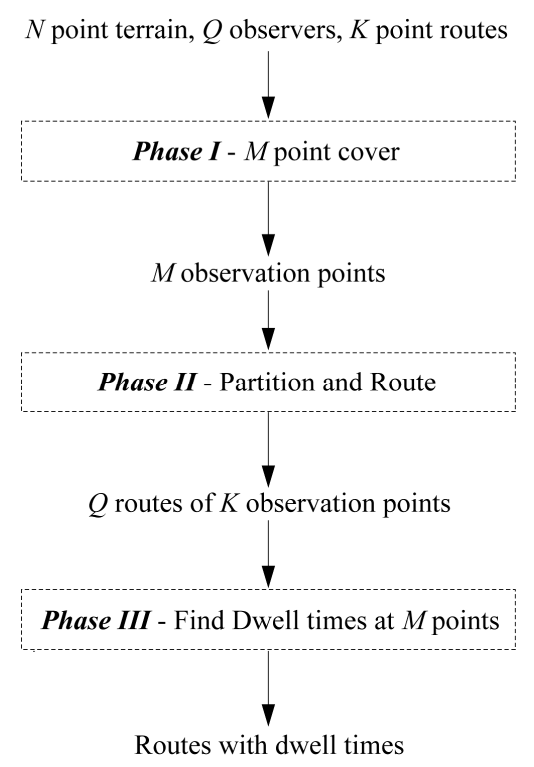

Figure 1. Flow chart of CF-PS methodology.

phase. Figure 1 provides a flowchart of steps of the CF-PS procedure.

\subsection{Phase $I-F i n d i n g ~ M$ Preferred Observation Points}

This problem is a special case of the SCP which is known as NP-hard. There are many covering heuristics for finding the best $M$ locations to place static observers (SCP heuristics). We considered four different covering heuristics referred to as: 1) COVER1, 2) COVER2, 3) COVERGA, and 4) COVERTIN. Each in turn is explained below.

\subsubsection{COVER1-Covering Heuristic 1}

COVER1 uses a greedy search, starting at the point with the largest cover, while covering continues to the next points, which will increase the total viewable area. Let $V_{i} \subseteq P$ represent the subset of points visible to $P_{i}$. Let $\left|V_{i}\right|$ represent the number of points within the subset $V_{i}$. Let $A \subseteq P$ represent the subset of preferred observation points. The objective of the heuristic is to find $|A|=M$ such that the total visible area to $A$ : $\bigcup_{P_{i} \in A} V_{i}$ will be maximized.

\section{COVER1 Algorithm}

Step 1: For every point $P_{i} \in P$ find $V_{i}$. Order the points in a descending order according to $\left|V_{i}\right|$ and starting from the top.

Step 2: Start with the current point $P_{i}$. Add $P_{i}$ to $A$ and delete it from the list. Go over the list of points and update $V_{i}$ for every point $P_{j}$ such that $V_{j}=V_{j}-V_{i}$. Reorder the list according to $\left|V_{j}\right|$. 
Step 3: Find the current point $P_{i}$. If $\left|V_{i}\right|>0$ and $|A|<M$ repeat step 2. Else, return $A$.

\subsubsection{COVER2-Covering Heuristic 2}

COVER2 starts with the greedy search and continues to the next best point, which is not visible from the previous selected points $[5,6]$.

\section{COVER2 Algorithm}

Step 1: For every point $P_{i} \in P$ find $V_{i}$. Order the points in a descending order according to $\left|V_{i}\right|$ and starting from the top.

Step 2: Start with the current point $P_{i}$. Add $P_{i}$ to $A$. Go over the list of points and delete every point visible to $P_{i}$ (including $P_{i}$ itself). If the list is not empty and $|A|<M$, repeat step 2. Else, return $A$.

\subsubsection{COVERGA-Covering Heuristic GA}

COVERGA - uses a genetic algorithm approach [13]. The GA approach for finding a set of $M$ observation points uses a chromosome encoding of length $2 M$, comprised of $M$ sequences of two genes, indicating the latitude and longitude coordinates of the selected observation points. Using the $3 \mathrm{D}$ visibility matrix $V$, we calculate the total number of points within the terrain which are visible to the $M$ observation points in the chromosome. This total number is used as the fitness value. Crossovers and mutations are allowed providing the gene values do not exceed the terrain's borders. Figure 2 shows the chromosome representation.

\subsubsection{COVERTIN-Covering Heuristic Using TIN}

When creating a TIN representation of the terrain out of a grid (height map) containing $n$ points, we select a subset of these points as vertices and connect them using edges to form a set of contiguous triangles. This representation replaces the original height map using only the data of the triangles and vertices. The height of the terrain represented by a TIN in a given point can be calculated using the plane equation of the triangle that covers it. The difference between the real height given by the grid and the interpolated height found using the TIN is called the error. When creating a triangulation out of a grid we can control the number of vertices and triangles using a specified maximal error (also called the level of

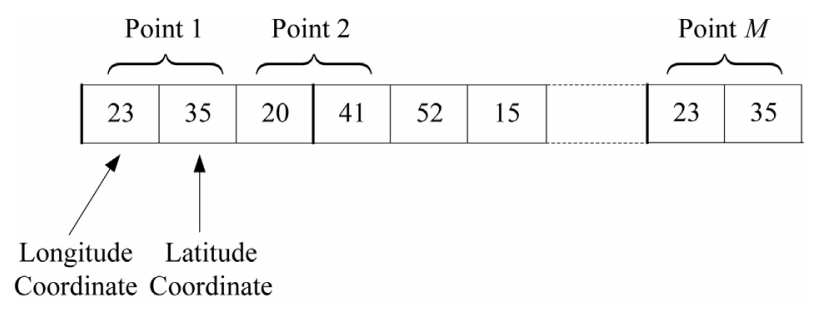

Figure 1. Chromosome representation. accuracy). Thus, a TIN representation with a small maximal error will contain many vertices and triangles as opposed to a TIN representation with a large maximal error.

In the COVERTIN heuristic $M$ selected observation points are the first $M$ points inserted as vertices when creating a TIN representation of the terrain, based on Garland and Heckbert's [14] triangulation for rendering a 3D terrain. Using this triangulation, vertices are inserted iteratively according to their error. Most of the first $M$ points found by this heuristics are peaks and saddle points, which are usually good for observation.

\subsection{Phase II-Creating $Q$ Routes using the Vehicle Routing Problem (VRP)}

The objective of this phase is to create a route for each of the $Q$ observers, which collectively pass through the $M$ observation points determined in Phase I, in minimum total time. This problem is solved using a modified version of the Vehicle Routing Problem (VRP). Note, that VRP is reducible to the TSP, and thus is also Np-Hard [12]. Many heuristics for solving the VRP are known, the most famous being the Savings Algorithm developed by Clarke \& Wright [12] which is employed here. We modify the standard VRP by allowing open end points, no demand quantities, no vehicle capacities, and add a requirement that adjacent points on the route exceed a minimum distance, $d$. Since the time horizon is fixed for each vehicle, minimizing travel time maximizes the allowable observation time (dwell time).

\subsection{Phase III-Assigning Dwell Times at Each Observation Point}

After creating the routes and the associated movement time, it is necessary to determine the dwell (stay) time at each of the occupied observation points. This problem is formulated as a linear programming (LP) problem, and is based on minimizing the maximal hit deviation from the average number of hits. We adopt the term hits to represent the "total number of hits per point", that is - the total number of discrete time units that a terrain point is visible collectively from all observers. The number of time units available is the residual time after removing travel times.

\subsubsection{Notation}

Let the points in the terrain be indexed $p=1,2, \cdots N$. Let $j$ represent the index of each observation point: $j=1$, $K,(K+1), 2 K,(2 K+1), 3 K,(M-K+1), M$. Of the $M=Q \cdot K$ points, the first $K$ points belong to the first observer; the next $K$ points to the second observer, and 
so on. $M T(q)$ denotes the total time spent on moving between observation points along route $q$. The residual time on route $q$ is $T(q)=T-M T(q)$. T( $q)$ is constant as $M T(q)$ is known from Phase II. Define $x_{j}$ as a decision variable representing the amount of the dwell time at observation point $j$ (which because of the indexing convention is the $[j-K \cdot([j / Q\rceil-1)]^{\text {th }}$ observation point along the $\lceil j / Q\rceil^{\text {th }}$ route). When solving the VACS problem, time is measured in small discrete units (meaning that $x_{j}$ must be an integer). However, since $T$ is large compared to the smallest time unit, $x_{j}$ is treated as a non negative continuous variable. We introduce the term "hit", for a point $p$, when an observer located at point $j$ has visibility indicator $v_{j, p}=1$ during one unit of time. Let the total hits at point $p$ be: $h_{p}=\sum_{j=1}^{M} x_{j} \cdot v_{j, p}$. Let $\bar{h}=\frac{1}{N} \sum_{p=1}^{N} h_{p}$ be the average number of hits over all points, and $L$ be its lower bound.

\subsubsection{LP Dwell Time Formulation for Phase III}

The LP approach has the objective of minimizing the largest deviation instead of the total deviation. This avoids the problem of having an uneven number of hits by smoothing out the size of the deviations. We define a new decision variable $E$, which indicates the largest deviation from the average number of hits. This is designed to provide a smooth visible coverage of the entire terrain.

Problem 2: Dwell Time LP

$$
\operatorname{Min} Z=E
$$

s.t.

$$
\begin{gathered}
x_{j} \leq S ; \forall j \\
\sum_{j=(q-1) \cdot K+1}^{q \cdot K} x_{j} \leq T(q) ; \forall q=1, \cdots Q \\
e_{p}^{+}+e_{p}^{-} \leq E ; \forall p \\
\sum_{j=1}^{M} x_{j} \cdot v_{j, p}+e_{p}^{+}-e_{p}^{-}=\frac{1}{N} \sum_{p=1}^{N} \sum_{j=1}^{M} x_{j} \cdot v_{j, p} ; \forall p \\
\frac{1}{N} \sum_{p=1}^{N} \sum_{j=1}^{M} x_{j} \cdot v_{j, p} \geq L
\end{gathered}
$$

$x_{j} \geq 0 \forall j ; \quad e_{p}^{+}, \quad e_{p}^{-} \geq 0 \forall p ; E \geq 0$.

The requirement that $x_{j}$, the dwell time, is bounded from above by $S$ leads to constraint (6). Constraint (7) insures dwell plus movement time along a single route must not exceed $T$. Let $e_{p}^{+}$and $e_{p}^{-}$respectively represent the under and over viewing error of point $p$ with respect to the average number of hits. As error values are complementary in any solution, constraint (8) insures that the minimal $E$ will equal the maximal deviation. Considering these deviations as non negative variables we obtain $h_{p}+e_{p}^{+}-e_{p}^{-}=\bar{h}$. After appropriate substitutions this can be fully written as (9). To avoid a solution in which all dwell times are set to zero, thus ensuring the minimal possible deviation, we add constraint (10) such that the average number of hits must exceed a given positive value $L$. The problem can be considered as a dual objective problem. Where the first priority is to maximize the average number of hits (this is in effect the same as the total number of hits), and the second priority is to minimize the largest deviation $E$ (which smoothes the visible coverage over the terrain). The solution procedure is to perform a right hand side ranging on constraint (10) by increasing $L$ until an infeasible solution is reached, and using this max value of $L$ solve Problem LP to minimize $E$ so as to smooth the coverage.

\subsection{Illustrative Example Using CF-PS Methodology}

To illustrate the methodology we use a small terrain, called the Dimona map, containing a $23 \times 21$ grid of 483 cells (considered as points), with each cell is of size $50_{m} \times 50_{m}$. The problem is to schedule two observers according to the parameters: 1) the planning horizon is 3600 seconds, 2) the observation time must not exceed 1200 seconds, 3) every observer must change its location 3 times, 4) the minimal distance between two consecutive observation points is $200 \mathrm{~m}$, and 5) the starting point is $\{0,0\}$. We solve the problem using four different sets of observation points (found by the four heuristics suggested in section 3.2).

\subsubsection{Solution}

Figure 3 graphically illustrates the four different solutions, each containing two routes passing through 6 observation points. The observation points in (a) and (b) are found using COVER1 and COVER2 heuristics respectively. The observation points in $(c)$ are found using GA, and those in $(d)$ are found using Garland and Heckbert's triangulation. The VRP heuristic was used on each set in order to determine the best two routes (for the two observers) that pass through all 6 points. The routes were found by the modified Clarke and Wright's savings algorithm for each of the four sets. The routes of observers A and $\mathrm{B}$ are marked using dashed white and black lines, respectively, with observation points indicated by the tags. Tables 1-4 record the coordinates of the observation points and associated dwell times of each. In addition the overall total travel and observation times of each observer are given. At the bottom of each table are the performance measures in terms of hits. 


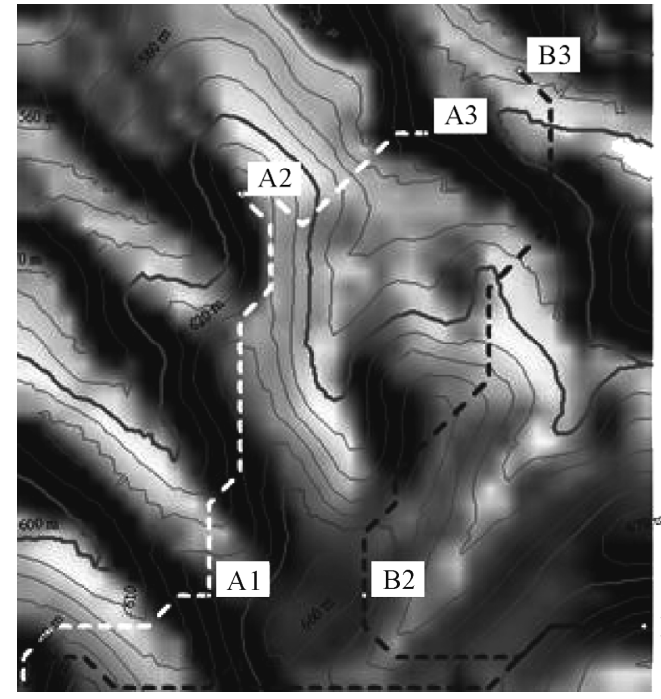

(a)

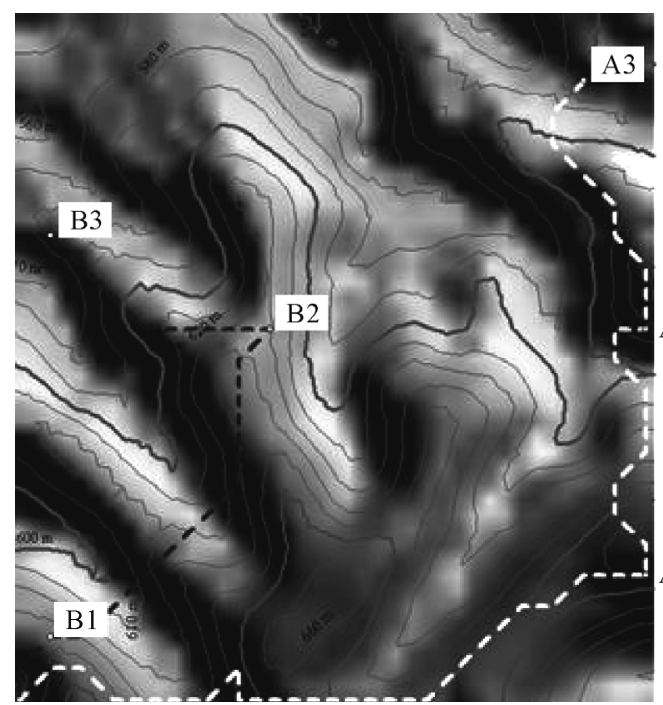

(c)

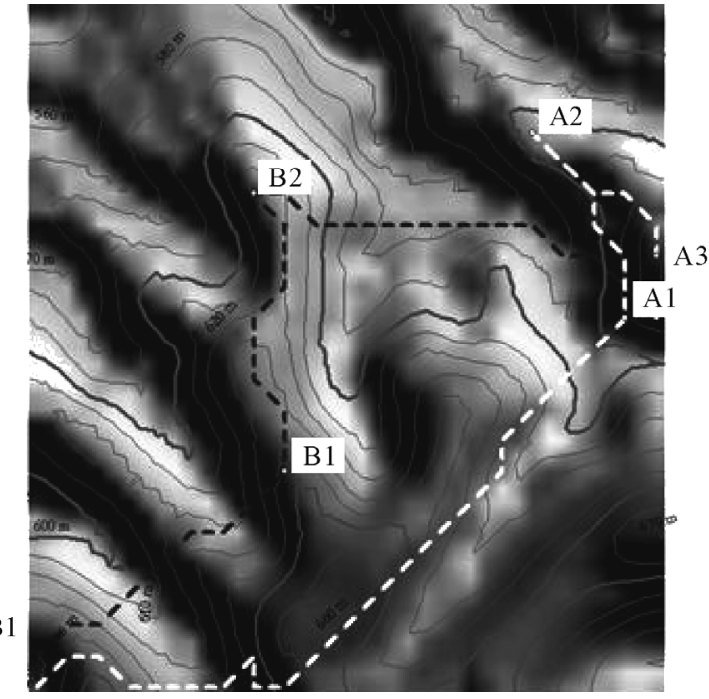

(b)

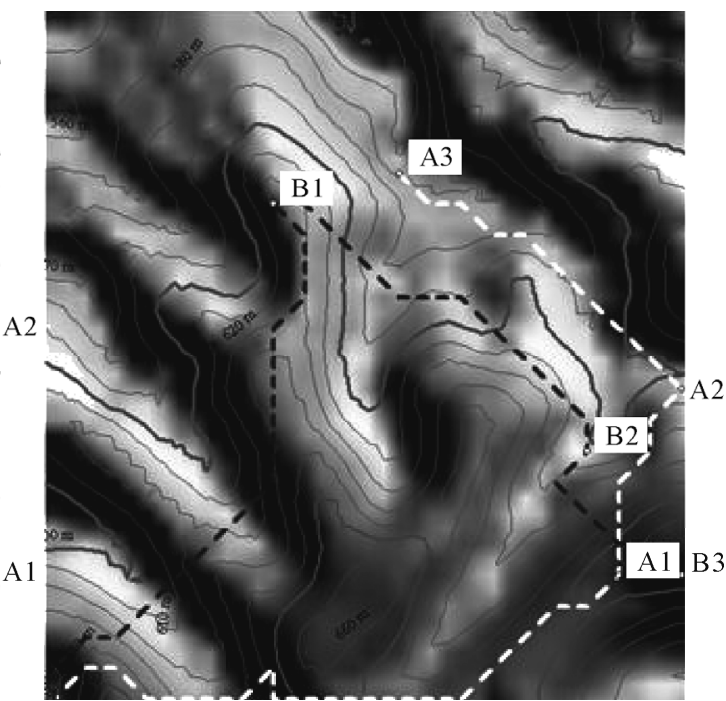

(d)

Figure 3. Final routes for CF-PS example, for two observers and four different initial covering points determined by: (a) COVER1, (b) COVER2, (c) COVERGA, and (d) COVERTIN. Observer A and B's locations are labeled A1-A3 and B1-B3 and connected with a white and black dashed lines, respectively.

\subsubsection{Discussion of Solution}

Tables 1-4 show that the total number of points hit in solutions $(a),(b)$, and $(d)$ is smaller than the total number of points hit in solution $(c)$. We can see that even though the total number of hits in solution $(c)$ is relatively large, its maximal deviation is the smallest. This implies that a good initial set of observation points (found in phase I), which together cover a large area of the terrain, is important when trying to reach a uniform hits distribution. Solutions $(a)$ and $(d)$ have a larger total travel time between observation points (opposed to solutions $(b)$ and (c)), leaving less time for observation. Thus, these solutions have much lower numbers of hits. Even though travel time takes a relatively small amount of the time horizon (approx 5\%) as opposed to dwell times (approx $95 \%$ ), the exchange of a unit of travel time for observer time has a very large impact on the number of hits (the area observed increases). Therefore it is important to find solutions with low travel time. Note that 100 percent coverage of all 483 points is not attained. Here using 6 observation points from 65 to 94 percent coverage is achieved. In section 5 it is shown that to achieve 100 percent coverage, from 12 to 37 observation points $(2.4$ to 7.7 percent of the total points in the terrain) are needed, depending on the covering heuristic used.

Though the above solutions are very different, they all 
Table 1. Sample solution using COVER1 for phase I-Figure 3(a).

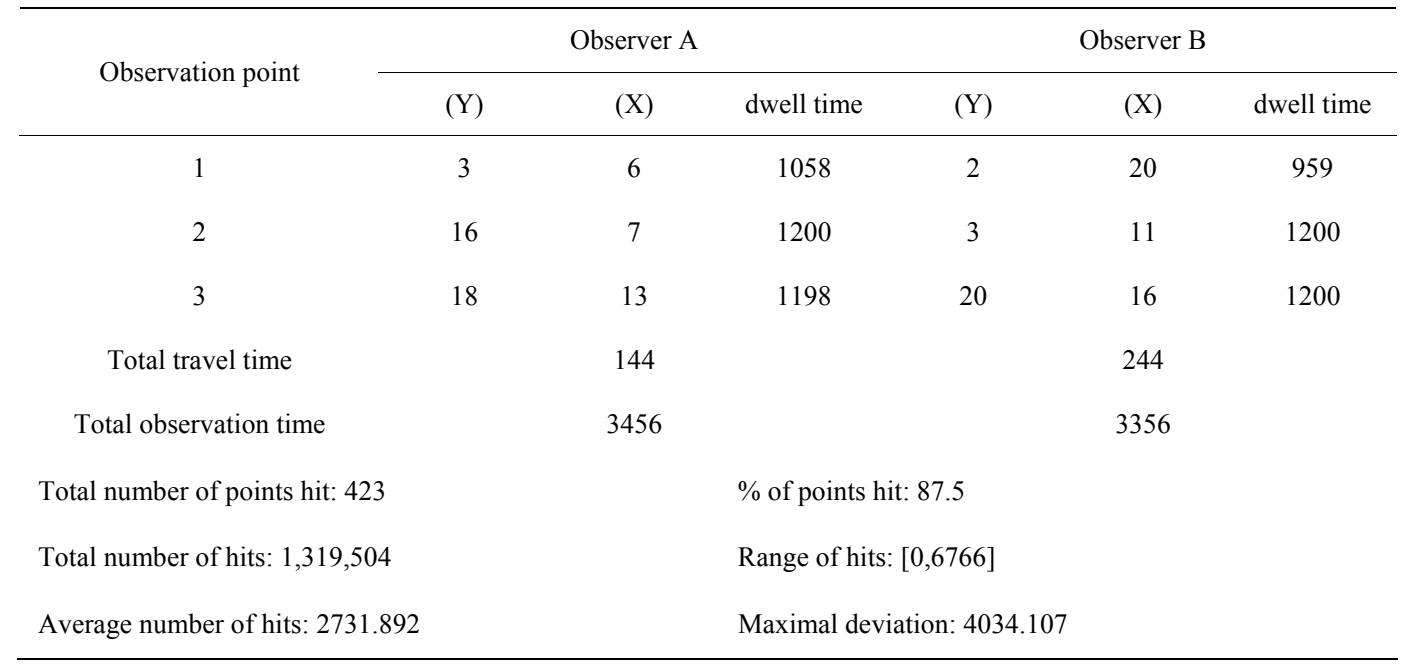

Table 2. Sample solution using COVER2 for phase I-Figure 3(b).

\begin{tabular}{|c|c|c|c|c|c|c|}
\hline \multirow{2}{*}{ Observation point } & \multicolumn{3}{|c|}{ Observer A } & \multicolumn{3}{|c|}{ Observer B } \\
\hline & $(\mathrm{Y})$ & $(\mathrm{X})$ & dwell time & $(\mathrm{Y})$ & $(\mathrm{X})$ & dwell time \\
\hline 1 & 12 & 19 & 1027 & 7 & 8 & 1200 \\
\hline 2 & 18 & 16 & 1200 & 16 & 7 & 1200 \\
\hline 3 & 14 & 20 & 1200 & 12 & 20 & 1027 \\
\hline Total travel time & & 173 & & & 173 & \\
\hline Total observation time & & 3427 & & & 3427 & \\
\hline \multicolumn{3}{|l|}{ Total number of points hit: 387} & \multicolumn{4}{|c|}{$\%$ of points hit: 80.1} \\
\hline \multicolumn{3}{|l|}{ Total number of hits: $1,899,424$} & \multicolumn{4}{|c|}{ Range of hits: $[0,6854]$} \\
\hline \multicolumn{3}{|l|}{ Average number of hits: 3932.554} & \multicolumn{4}{|c|}{ Maximal deviation: 3932.554} \\
\hline
\end{tabular}

Table 3. Sample solution using COVERGA for phase I-Figure 3(c).

\begin{tabular}{|c|c|c|c|c|c|c|}
\hline \multirow{2}{*}{ Observation point } & \multicolumn{3}{|c|}{ Observer A } & \multicolumn{3}{|c|}{ Observer B } \\
\hline & $(\mathrm{Y})$ & $(\mathrm{X})$ & dwell time & $(\mathrm{Y})$ & $(\mathrm{X})$ & dwell time \\
\hline 1 & 4 & 20 & 1200 & 2 & 1 & 1084 \\
\hline 2 & 12 & 20 & 1200 & 12 & 8 & 1200 \\
\hline 3 & 20 & 18 & 991 & 15 & 1 & 1200 \\
\hline Total travel time & & 209 & & & 116 & \\
\hline Total observation time & & 3391 & & & 3484 & \\
\hline \multicolumn{3}{|l|}{ Total number of points hit: 457} & \multicolumn{4}{|c|}{$\%$ of points hit: 94} \\
\hline \multicolumn{3}{|l|}{ Total number of hits: $1,535,205$} & \multicolumn{4}{|c|}{ Range of hits: $[0,6875]$} \\
\hline \multicolumn{3}{|l|}{ Average number of hits: 3178.478} & \multicolumn{4}{|c|}{ Maximal deviation: 3696.521} \\
\hline
\end{tabular}


Table 4. Sample solution using COVERTIN for phase I - Figure 3(d).

\begin{tabular}{|c|c|c|c|c|c|c|}
\hline \multirow{2}{*}{ Observation point } & \multicolumn{3}{|c|}{ Observer A } & \multicolumn{3}{|c|}{ Observer B } \\
\hline & $(\mathrm{Y})$ & $(\mathrm{X})$ & dwell time & $(\mathrm{Y})$ & $(\mathrm{X})$ & dwell time \\
\hline 1 & 4 & 18 & 1200 & 16 & 7 & 1200 \\
\hline 2 & 10 & 20 & 1016 & 8 & 17 & 1004 \\
\hline 3 & 17 & 11 & 1200 & 4 & 20 & 1200 \\
\hline Total travel time & & 184 & & & 196 & \\
\hline Total observation time & & 3416 & & & 3404 & \\
\hline \multicolumn{3}{|c|}{ Total number of points hit: 315} & \multicolumn{4}{|c|}{$\%$ of points hit: 65.2} \\
\hline \multicolumn{3}{|c|}{ Total number of hits: $1,091,393$} & \multicolumn{4}{|c|}{ Range of hits: $[0,6811]$} \\
\hline \multicolumn{3}{|c|}{ Average number of hits: 2259.612} & \multicolumn{4}{|c|}{ Maximal deviation: 4551.387} \\
\hline
\end{tabular}

show a repeating pattern. Each observer focuses on a different part of the area. This strengthens the idea that another methodology can be applied here as well. This method would first divide the area between the observers (such that each one will have its own region), and then create a route for each observer within its own region. This method starts with partitioning and then finds a covering route for each region, and therefore, it is classified as "partition first, cover second". This solution methodology is discussed in the next section.

\section{The Partition First, Cover Second (PF-CS) Heuristic}

\subsection{Overview}

This methodology contains three phases, starting with a terrain triangulation of a given resolution, partitioning it into $Q$ regions, one for each observer. In phase I area partitioning is done starting with a triangle irregular networks (TINs), where the basic primitives are the triangles. Initial creation of this triangulation is done using Garland and Heckbert's Algorithm [14] and is based on a desired resolution level. We create a partitioning of the area into $Q$ sub areas (comprised of contiguous triangles), one for each observer. In a second phase, $K$ points in each region are found which provide the maximum visible cover and then a route for every region is created. The observation times at each point on the routes are determined using a LP in the third phase. Once there are $Q$ routes, we solve LP dwell time problem to find the dwell times for each observer at each observation point. Figure 4 provides a flowchart of the methodology.

\subsection{Phase I-Area Partitioning}

Partitioning starts with a given TIN representation of the

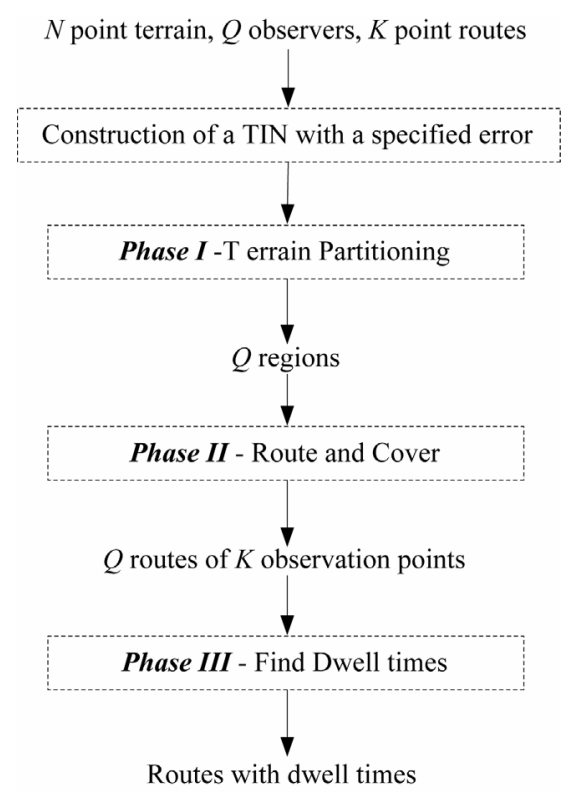

Figure 4. Flow chart of the PF-CS methodology.

terrain, with $n$ triangle primitives, denoted as $T$. Triangles are combined to form a "Q-partition". A $Q$-partition of $T$ is comprised of $Q$ subsets $T_{1}, \cdots, T_{i}, \cdots, T_{Q}$ of $T$ such that; $U T_{i}=T$ and $T_{i} \cap T_{j}=\varphi \quad \forall i, j$. The area partition problem is to find a $T$-partition of $T$ such that the areas of subsets $T_{i}$ are close to equal. In addition, all the triangles in $T_{i}$ are contiguous, such that any two triangles share a common side. A $Q$-partition may be obtained through an iterative process, starting with $n$ triangles and joining contiguous triangles until there are $Q$ continuous, non-empty regions such that each region contains a finite number of triangles from the original TIN. To cover a region containing $n$ vertices, at most $[(n+1) / 2]$ of its vertices are used. Figure 5(a) shows a narrow region containing 18 triangles. Note, that only the vertices 2, 4, 6 and 8 are needed to cover this region. Figure 5(b) 


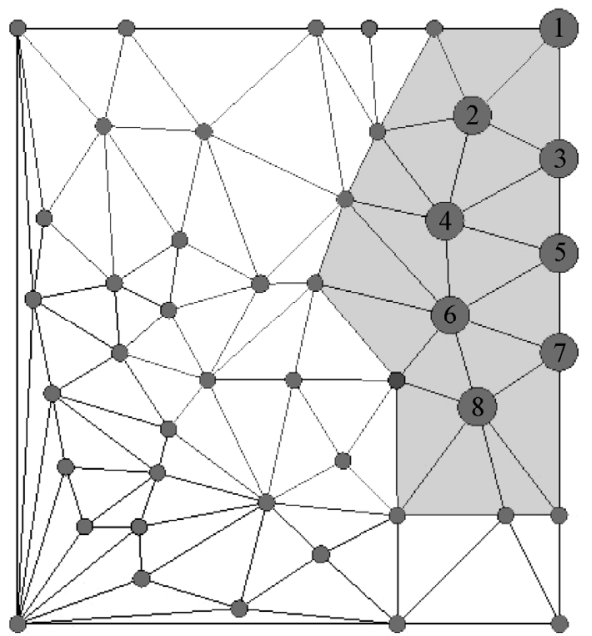

(a)

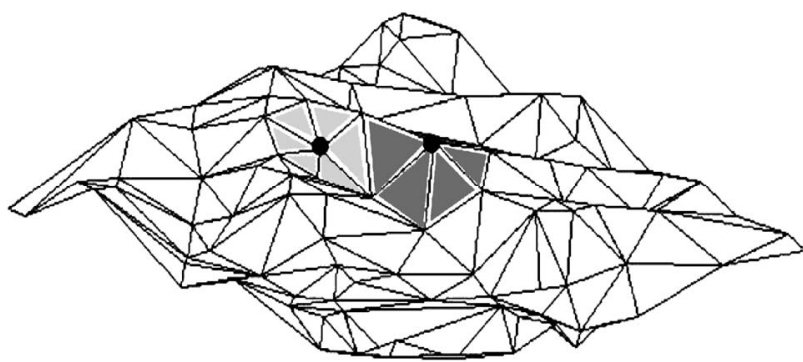

(b)

Figure 5. (a) A narrow region; (b) The use of vertices as observation points.

shows a 3D TIN representation of a small map containing 70 vertices. The partition problem is NP-Hard even when only two regions with the same area are allowed [15], and therefore a heuristic is needed. However, for small problems a binary integer formulation is proposed.

\subsubsection{Binary Integer Linear Programming Formulation of the Balanced Area Partition (BAP) Problem}

The balanced area partition problem can be formulated as a $0-1$ integer programming problem by defining binary variables $x_{i, j}=1$ if triangle $j(j=1, \cdots, n)$ is assigned to $T_{i}(i=1, \cdots, Q)$, and 0 otherwise. Let $a_{j}$ represent the area of triangle $j, A_{i}$ the area of $T_{i}$, such that $A_{i}=\sum_{j} a_{j} x_{i, j} ; \forall i$ and $A$ the area of $T$. Let $T A(i, j)$

be a triangle adjacency indicator, equal to 1 if triangles $i$ and $j$ are adjacent, and 0 otherwise. The following is a formulation for minimizing the largest deviation $E$ from the balanced area state $A / Q$ of the $Q$-partition.

Problem 3: Balanced area partition integer program

$$
\operatorname{Min} Z=E
$$

s.t.

$$
\begin{gathered}
\sum_{j=1}^{n} a_{j} x_{i, j}+e_{i}^{+}-e_{i}^{-}=A / Q ; \forall i \\
e_{i}^{+}+e_{i}^{-} \leq E ; \forall i \\
x_{i, j} \leq \sum_{k=1}^{n} T A(j, k) x_{i, k} ; \forall i, j \\
\sum_{i}^{Q} x_{i, j}=1 ; \forall j
\end{gathered}
$$

$X_{i, j}=\{0,1\} \forall i, j ; \quad e_{i}^{+}, e_{i}^{-} \forall i ; \quad E \geq 0$.

Note that $e_{i}^{+}, e_{i}^{-}$are the deviation errors which can be shown to be complimentary. Constraint (11) compares the target area with the actual area of each partition element $T_{i}$. Constraint (12) states that the deviation error is below the max error $E$. Constraint (13) states that if a triangle $j$ is assigned to $T_{i}$ then it must be adjacent to at least one of the other triangles assigned to $T_{i}$. This insures that the set of triangles in any $T_{i}$ are contagious. Constraint (14) insures that each triangle $j$ is assigned to exactly one triangular subset $T_{i}$. Note that the problem does not consider the "compactness" of the triangular subsets, and thus long narrow subsets may be obtained. Because integer nonlinear programming problems require long solution times we propose the following fast heuristic to determine the Q-partition.

\subsubsection{A Greedy Algorithm for the Balanced Area Partitioning (BAP) Problem}

The greedy area partitioning heuristic starts with a single vertex, creates a region from all the triangles attached to that vertex, and iteratively adds vertices to that region until either of two conditions is violated. When this occurs a new region is established. The region growing stopping conditions are: 1) the area of the region must not exceed the area of the terrain divided by the number of observers (balanced area), and 2) the distance between the two furthest points within each region must not exceed a predefined distance (this avoids long narrow regions). The triangulation $T$ is represented as a graph $G=\{V, \Gamma\}$ where, $V$ is the set of vertices, and $\Gamma$ is the set of arcs. Let $N A$ be a node adjacency matrix $n \times n$ matrix with common element $\alpha_{i, j}=1$ if vertices $i$ and $j$ are adjacent (both vertices appear in the same triangle), and 0 otherwise. Let $V_{t i}$ represent a set of all triangles connected to vertex $i$. Let $A\left(V_{i}\right)$ represent the total area of the triangles attached to vertex $i$. The BAP algorithm can be shown to be polynomial of order $O\left(n^{2}\right)$.

\section{The BAP algorithm}

1) Calculate $A\left(V_{i}\right)$ for every vertex $i \in V$. Sort the vertices in descending order of $A\left(V_{i}\right)$. Let $j=1$ and let $c$ be the first vertex in the list.

2) Add vertex $c$ to region $T_{j}$. a) Delete vertex $c$ from 
the list. b) For every vertex $i \in V$, if $V_{t c} \cap V_{t i} \neq \phi$, decrease the common triangle's areas out of $V_{A i}$ such that; $A\left(V_{i}\right)=A\left(V_{i}\right)-A\left(V_{t i} \cap V_{t c}\right), \forall i$. c) Sort the list of the remaining vertices $V$ in decreasing order according to $A\left(V_{i}\right)$. d) Find the next vertex $c$ in $V$ which is a neighbor of one of the vertices in the current region $T_{j}$, and adds the largest area to the region, i.e. find a vertex $c$ such that, $c=\arg \operatorname{Max}\left\{A\left(V_{i}\right) \mid \alpha_{i, k}=1 \forall v_{k} \in T_{j}\right\}$.

3 ) If at least one of the above mentioned two conditions is violated, then a new region is established. Let $j=j+1$. Find $c$, among unassigned vertices, with the max area: $c=\arg \operatorname{Max}\left\{A\left(V_{i}\right) \mid v_{i} \in V\right\}$. Go to step 2 .

4) Repeat steps 2 and 3 until $V=\phi$.

\subsection{Phase II-Finding Covering Routes}

The partition of the region provides $Q$ smaller problems. In each we need to locate only $K$, and not $M=Q \cdot K$ observation points using the COVER1 heuristic (see section 3.2.1), and then solve a single vehicle routing problem instead of the $Q$ vehicle routing problem as in the previous approach. The routing problem becomes one of a simple shortest path problem through $K$ points with a given start point and an open end point.

\subsection{Phase III-Assigning Dwell Times}

After creating the routes, the LP dwell time problem is solved.

\subsection{Illustrative Example Using PF-CS Methodology}

To illustrate the methodology we use the same terrain presented in Figure 3 and the same assumptions of the CF-PS example. Phase I uses the greedy BAP heuristic over a triangulation with a $5 \mathrm{~m}$ level of accuracy. In phase II the COVER1 heuristic is used in each region to find the location of the 3 observation points, followed by the use of the modified savings algorithm to find the route through each set of points in each region. Phase III uses the dwell time LP in order to determine the observation time at each observation point.

\subsubsection{Solution}

After performing the partitioning into two regions we get the two regions shown in Figure 6(a). Observer $A$ is assigned to the area colored gray and observer B is assigned to the area colored white. To help the reader visualize the triangulation, a picture of the terrain from the same view point is given in Figure 6(b). The covering points found and the two routes through them are shown in Figures 6(c) and (d). The labels in the figures show the covering points, and the routes of observers A and B are indicated by the dashed white and black lines, respectively. Table 5 shows the coordinates of the observation points and their associated dwell times. In addition the total travel and observation times of each observer are given. At the bottom of each table are the performance measures in terms of hits.

\subsubsection{Discussion of Solution}

The terrain was divided into the two regions presented in Figure 6(a), assigning observer $A$ to the north-western region, and observer $\mathrm{B}$ to the eastern region. Table 5 shows that the total number of points hit in this solution (and therefore the $\%$ of area covered) is better than in solutions 6(a), (b), and (d) for the previous CF-PS example, but yet its maximal deviation is larger. One can see that the dwell LP worked as it did seem to push down the deviation above the mean $6859-4075=2784$. The $\max$ dev of 4075 is the same as the mean, meaning it occurred

Table 5. A sample solution using the area partitioning methodology.

\begin{tabular}{|c|c|c|c|c|c|c|}
\hline \multirow{2}{*}{ Observation point } & \multicolumn{3}{|c|}{ Observer A } & \multicolumn{3}{|c|}{ Observer B } \\
\hline & $(\mathrm{Y})$ & $(\mathrm{X})$ & dwell time & $(\mathrm{Y})$ & $(\mathrm{X})$ & dwell time \\
\hline 1 & 10 & 13 & 1200 & 8 & 1 & 1200 \\
\hline 2 & 13 & 20 & 1200 & 15 & 1 & 1066 \\
\hline 3 & 4 & 20 & 993 & 16 & 8 & 1200 \\
\hline Total travel time & & 207 & & & 134 & \\
\hline Total observation time & & 3393 & & & 3466 & \\
\hline Total number of points hit: 436 & & & \multicolumn{4}{|c|}{$\%$ of points hit: 90.2} \\
\hline Total number of hits: $1,968,430$ & & & \multicolumn{4}{|c|}{ Range of hits: $[0,6859]$} \\
\hline Average number of hits: 4075.424 & & & \multicolumn{4}{|c|}{ Maximal deviation: 4075.424} \\
\hline
\end{tabular}




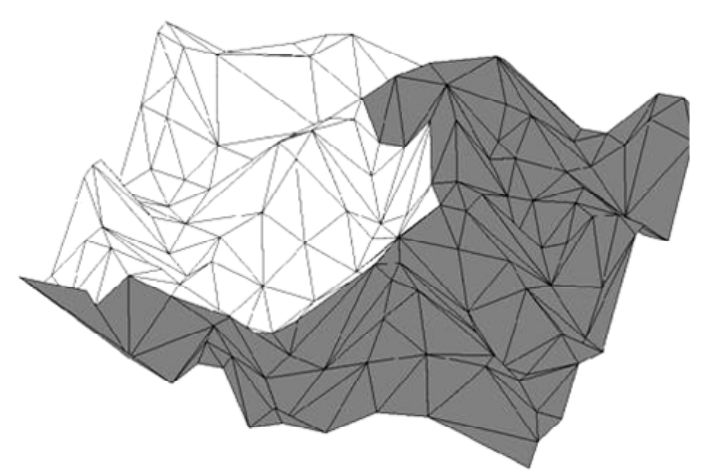

(a)

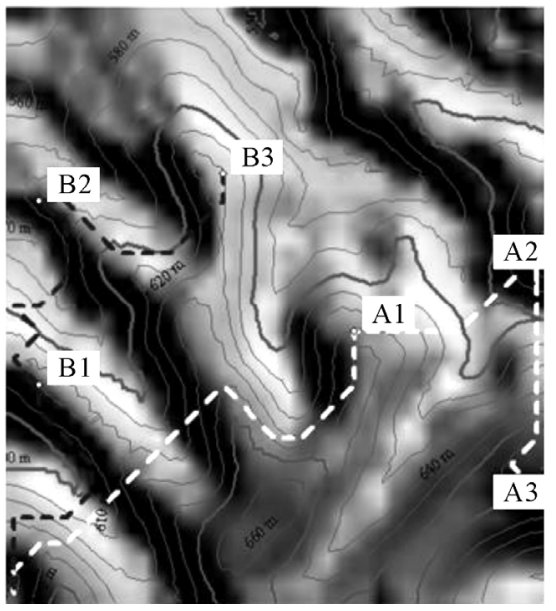

(c)

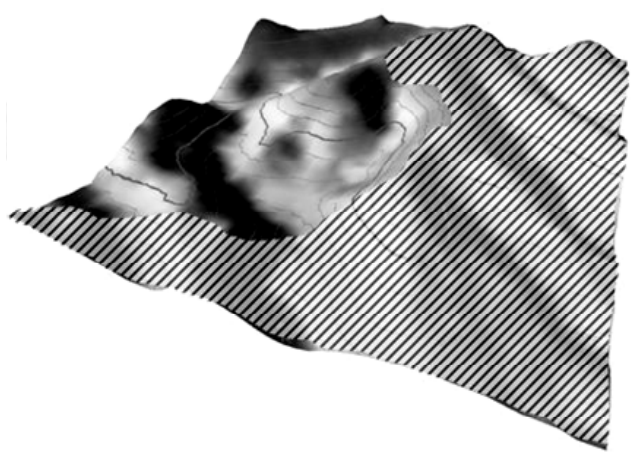

(b)

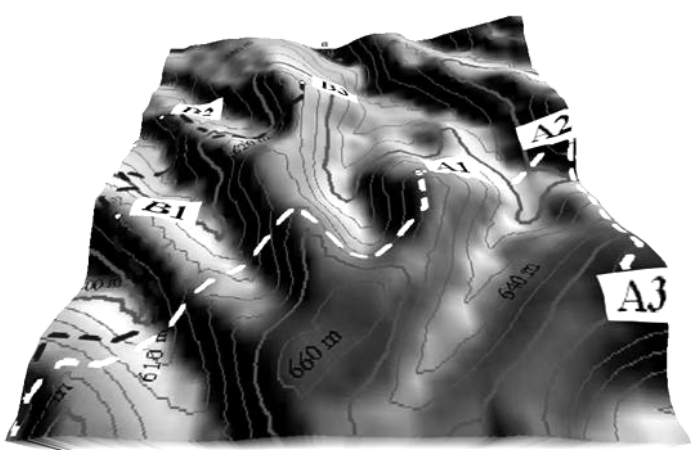

(d)

Figure 6. Partitioning of the map into two regions, and the resultant routes for PF-CS example. (a) TIN representation of the terrain and its partition into two regions; (b) another view of the partition; (c) final routes and observation points; (d) 3D visualization of the solution.

between 0 hits (points not seen) and the mean 4075. This is because the mean was very high (each point was seen 4075 times or seconds on average) which is good, as it implies the total number of hits was also very high.

\section{Performance Comparison of CF-PS and PF-CS Decomposition Methods}

\subsection{Test Parameters}

Several tests were conducted to compare the quality of the solutions achieved for the VACS problem using both CF-PS and PF-CS decomposition heuristics. The tests examined the solutions found by the methodologies for three maps and two scenarios, using 4 and 3 different versions of the CF-PS and PF-CS methods, respectively. This resulted in a total of 42 test cases. The performance of both methodologies was evaluated by function (1), which represented the total visibility of the terrain by all observers, penalized for multiple coverage. Three types of maps, differing by size and topographic structures, were tested; 1) Dimona map, grid $=23 \times 21$, terrain area $=1 \times 1 \mathrm{~km}, 2)$ Modiin map, grid $=61 \times 61$, terrain area $=$ $3 \times 3 \mathrm{~km}, 3$ ) Valley of Elah map, grid $=81 \times 82$, terrain area $=4 \times 4 \mathrm{~km}$. Figure 7 shows the terrains, where contour lines are 10 meters apart. The various grid sizes represent up to a 400 Percent scale up of the problem size. For the CF-PS method the original rectangular grid point height representation of the terrain is used. Four covering versions are tested (COVER1, COVER2, COVERGA and COVERTIN) for this method. For the PF-CS method the grid height maps are converted to TIN representations. Three different TIN versions are tested, each representing different approximations of the terrain. These are denoted as TIN5, TIN10 and TIN20 for approximations of $5 \mathrm{~m}, 10 \mathrm{~m}$, and $20 \mathrm{~m}$ levels of accuracy.

Observation missions can have different objectives according to different scenarios. This can have a large effect on the desirable number of relocations and the allowable observation dwell times. We chose to examine the two following scenarios: 


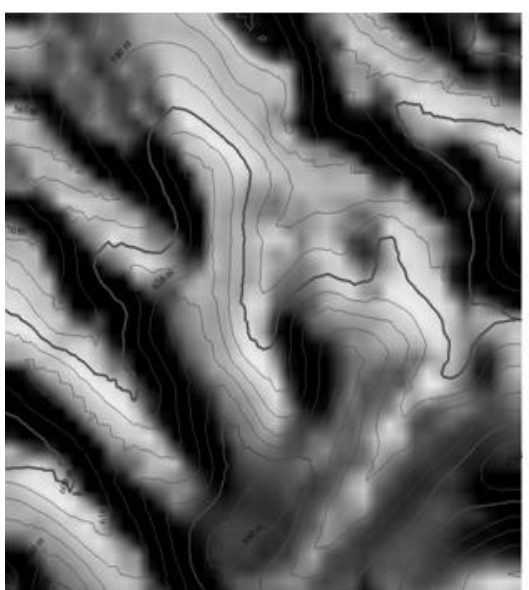

(a)

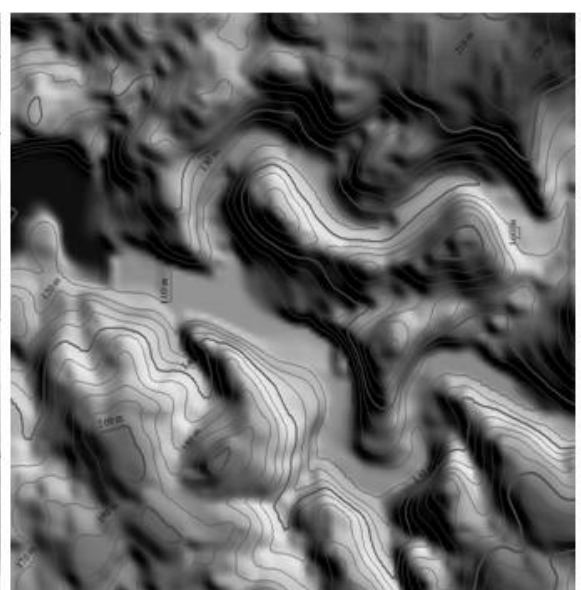

(b)

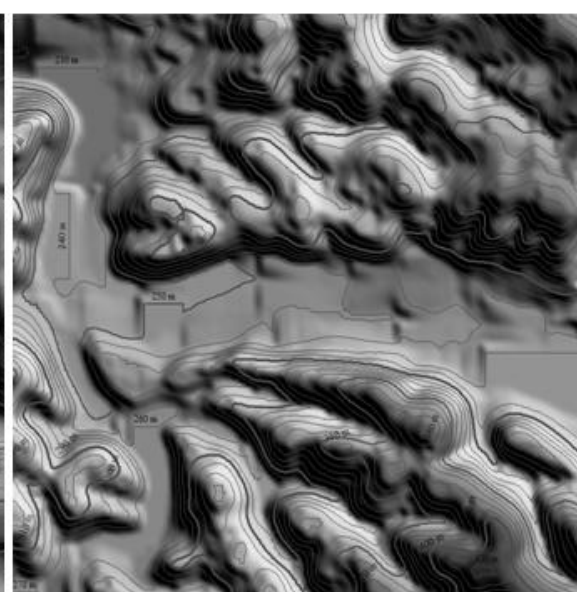

(c)

Figure 7. 3D contour maps of the three tested terrains.

First scenario: Observation teams are sent to enemy territory to locate an object (such as a crashed aircraft). The observers must visibly cover the entire terrain while spending limited time at any location. Every point must be viewed just enough to determine if the object exists. An observer must move so the distance between locations exceeds a minimum distance to avoid detection. This type of scenario dictates the use of many observation points (for complete coverage) without spending too much time in each. Thus, the maximal dwell time $S$ at every observation point is relatively small.

Second scenario: Several observation teams are in enemy territory looking for rocket launchers. Rockets can be launched from almost any point within the terrain at any time. Since rocket locations are unknown, it is desirable for observers to use large coverage points for quick detection of launchers. Here also the team's moves must exceed a minimum distance. This type of scenario dictates the use of fewer observation points spending a relatively large time at each, such that large areas will be visible. The maximal dwell time $S$ should be relatively large.

Table 6 presents the values for; $Q$ - the number of observers for each map, $K$ - the number of observation points for each route, $S$ - the maximal allowed observation dwell time in seconds at each observation point, $d$ the minimal distance between every two consecutive points, and $T$ - the planning horizon. Note that more observation points were used for the larger sized terrains and for the first scenario.

In addition, a sensitivity analysis was made (using the CF-PS method, and the Dimona map) to study the affect of the number of observation points on the percent of area (terrain points) covered. Finally, all of results were subjectively evaluated by soliciting the opinion of several experts in the field.

\subsection{Test Results}

Table 7 presents the test results using CF-PS and PF-CS methodologies (values $=1^{\text {st }}$ scenario $/ 2^{\text {nd }}$ scenario). Since the 3 versions of the PF-CS correspond to three TIN approximations with a different number of triangles and vertices, and consequently a different partitioning different solutions were obtained. The performance value was calculated using the objective of the VACS problem (Equation (1)). This function sums the total number of viewable points within the terrain to all observers (not including overlaps) throughout the entire planning horizon, giving an exponentially decreasing contribution (starting from 1 and going to 0 ) to each visible point according to the number of times it was previously seen.

\subsection{Two Sample Solutions}

Figure 8 illustrates the solution using both methodologies for a $16 \mathrm{~km}^{2}$ terrain of the Valley of Elah (the area in which David confronted Goliath). There are three observers, each changing its location 3 times. The labels indicate the order of visits of each observer and their observation time (sec.). Figure 8(a) represents the solution achieved by the CF-PS methodology using COVERGA for phase I. Figure 8(b) represents the solution achieved by the PF-CS methodology based on a TIN with a $5 \mathrm{~m}$ level of accuracy.

\subsubsection{CF-PS (Figure 8(a))}

The set of covering points was selected using GA (Phase I) which was found to give good visual coverage. In Phase II the routes where generated using the modified savings algorithm In Phase III the observation dwell times where determined by solving problem LP. The code was implemented in JAVA and the LP was solved 
Table 6. Test parameters for all maps and scenarios (table values are $1^{\text {st }}$ scenario/ $2^{\text {nd }}$ scenario).

\begin{tabular}{cccccc}
\hline Map & $\boldsymbol{Q}$ & $\boldsymbol{K}$ & $\boldsymbol{S}$ & $\boldsymbol{d}$ & $\boldsymbol{T}$ \\
\hline Dimona & $2 / 2$ & $5 / 3$ & $600 / 1200$ & $200 / 200$ & $3600 / 3600$ \\
Modiin & $3 / 2$ & $5 / 4$ & $600 / 1800$ & $400 / 400$ & $7200 / 7200$ \\
Valley of Elah & $3 / 3$ & $8 / 3$ & $600 / 2400$ & $500 / 500$ & $7200 / 7200$ \\
\hline
\end{tabular}

Table 7. Results for CF-PS and PF-CS (col 4 and 5 values are for $1^{\text {st }}$ scenario/ $2^{\text {nd }}$ scenario).

\begin{tabular}{|c|c|c|c|c|}
\hline \multirow[t]{4}{*}{ Map } & Method & Version & No. observers, No. points & Performance Value* 10 \\
\hline & \multirow{4}{*}{ CF-PS } & COVER1 & $2.10 / 2.6$ & $21.6 / 51$ \\
\hline & & COVER2 & $2.10 / 2.6$ & $19.1 / 50.6$ \\
\hline & & COVERGA & $2.10 / 2.6$ & 24.5/61.6 \\
\hline \multirow[t]{7}{*}{ Dimona } & & COVERTIN & $2.10 / 2.6$ & $14.3 / 29.4$ \\
\hline & & TIN 20 & $2.10 / 2.6$ & $23.4 / 60.5$ \\
\hline & PF-CS & TIN 10 & $2.10 / 2.6$ & $23.5 / 62.8$ \\
\hline & & TIN 5 & $2.10 / 2.6$ & 23.8/62.9 \\
\hline & & COVER1 & $3.15 / 2.8$ & $197.7 / 484.2$ \\
\hline & $\mathrm{CD} D \mathrm{DC}$ & COVER2 & $3.15 / 2.8$ & $236.9 / 527.3$ \\
\hline & & COVERGA & $3.15 / 2.8$ & 292.7/716.5 \\
\hline \multirow[t]{7}{*}{ Modiin } & & COVERTIN & $3.15 / 2.8$ & $186.6 / 358.2$ \\
\hline & & TIN 20 & $3.15 / 2.8$ & $275 / 645.5$ \\
\hline & PF-CS & TIN 10 & $3.15 / 2.8$ & $290 / 673.4$ \\
\hline & & TIN 5 & $3.15 / 2.8$ & $285.6 / 642.9$ \\
\hline & & COVER1 & $3.24 / 3.9$ & $409.6 / 1426$ \\
\hline & & COVER2 & $3.24 / 3.9$ & $423.5 / 1451$ \\
\hline & & COVERGA & $3.24 / 3.9$ & $569.6 / 2024$ \\
\hline \multirow[t]{4}{*}{ Valley of Elah } & & COVERTIN & $3.24 / 3.9$ & $507.3 / 1810$ \\
\hline & & TIN 20 & $3.24 / 3.9$ & $555.9 / 1896$ \\
\hline & PF-CS & TIN 10 & $3.24 / 3.9$ & $566.3 / 1946$ \\
\hline & & TIN 5 & $3.24 / 3.9$ & $566.2 / 1946$ \\
\hline
\end{tabular}

using Matlab. This solution produces a clear area partitioning between the observers. Observer $\mathrm{C}$ moves along the western ridge (advancing from south to north), which visually covers the entire valley and most of the gorges which flow from east to west. Observer B covers the southern part of the terrain moving from west to east. Observer A covers the northern side of the terrain with its deep gorges, which are not visible from the western/ southern ridge.

\subsubsection{PF-CS (Figure 8(b))}

In Phase I the area was partitioned to the three regions for a terrain triangulation of $5 \mathrm{~m}$ accuracy. In Phase II the observation points in each region were selected using a greedy SCP heuristic, and the routes which pass through them were generated using the shortest path algorithm. Phase III also used the LP problem. In this solution we can see once again that each observer focuses on a restricted region. Observer A is sent to the north-western 


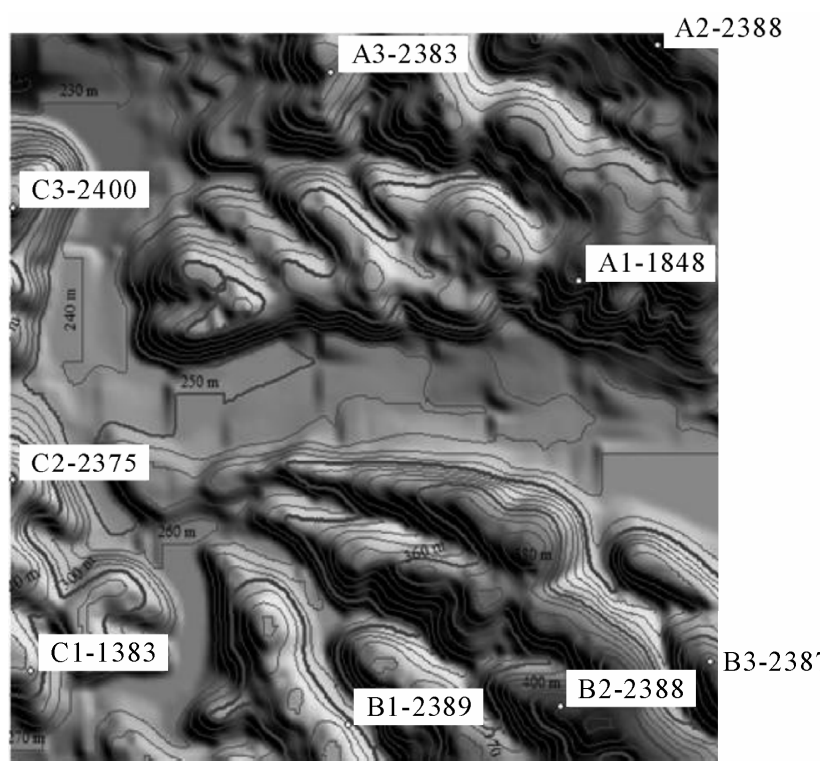

(a)

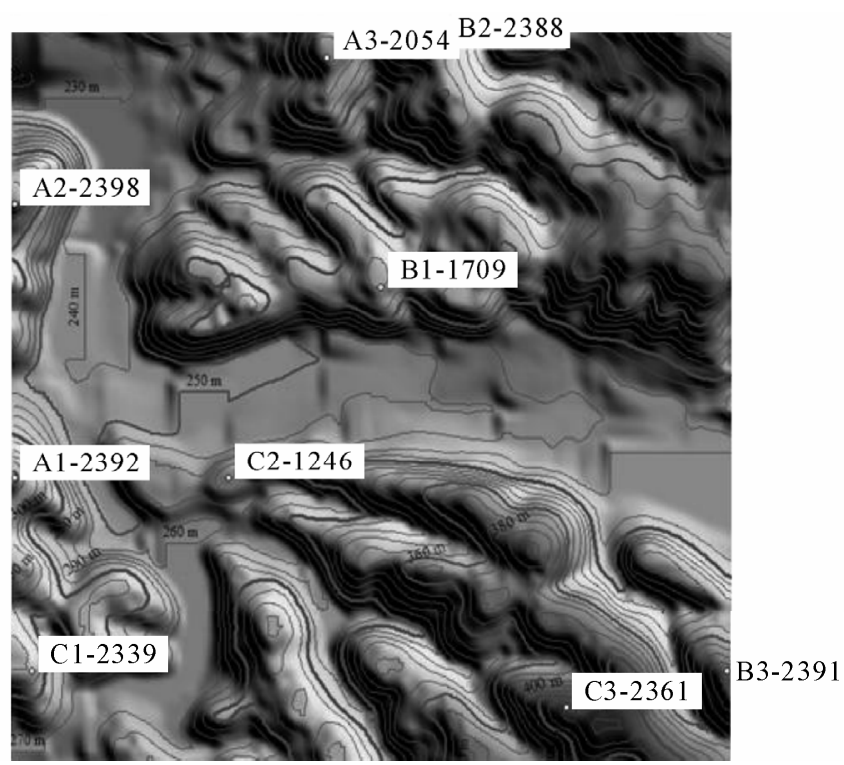

(b)

Figure 8. Two sample solutions using: (a) CF-PS , (b) PF-CS.

part, Observer B is responsible on the eastern part, and observer $\mathrm{C}$ is sent to the southern part.

\subsection{Sensitivity to the Number of Observation Points}

We also examined the effect of the size of the subset of candidate observation points, $M$, taken from all 483 points of the grid representation of the Dimona map on the total percent of terrain grid points seen at least once (percent of area covered by the observation points). This was done using the four covering heuristics of CF-PS method while varying the number of observation points $M$ from 1 to 483 as shown in Figure 9.

\subsection{Expert Opinion}

Solutions were presented to two military experts, who where responsible for placing observation teams in combat zones. Both indicated the solutions included excellent observation points and routes providing good coverage, and that computerized methodologies were very helpful. They indicated that determining the location of observation points and the routes between them is time consuming when done manually, and until now there had not been a method to establish the quality of a solution.

\subsection{Discussion of Results}

Results using three terrains of various grid sizes represented up to a 400 Percent scale up of the problem size.
Comparison of the performance values across problem size is not valid as the values are inflated for larger size terrains. The results show that for the CF-PS method (Table 7) the Genetic Algorithm covering approach resulted better performance for all scenarios and maps. The computation times, however, were considerably larger for this approach. This is because it is guided by a total area coverage objective, and maintaining a population of solutions at each iteration. For the PF-CS method, performance varies depending on the terrain, but in general finer resolutions of the TIN resulted in higher performance. Independent of the method, the $2^{\text {nd }}$ scenario performance times are larger than those in the $1^{\text {st }}$ scenario for the Dimona and Modiin maps. This is expected because the allowable dwell time at the observation points are 2 to 3 times longer than in the $1^{\text {st }}$ scenario, so the good visibility positions provide more hits on each visible point. This was not true, however, for the Valley of Elah map. The many irregularities defeated the advantages of long dwell times, as there were fewer opportunities to view large areas from single observation points. When comparing the best solutions of both methods the CF-PS method was slightly better. However, because of the increased computation time we suggest that the PF-CS method with the finer terrain TIN approximation be used. This method is faster as partitioning the terrain into separate regions for each observer, provides smaller coverage and routing problems. The GA version took from 13 to 180 minutes to run, while all other methods took from 4 to 20 minutes. The code for all methodologies was written in Visual Java 6, and was not optimized. All calculations were performed on a Pentium 4 PC with 


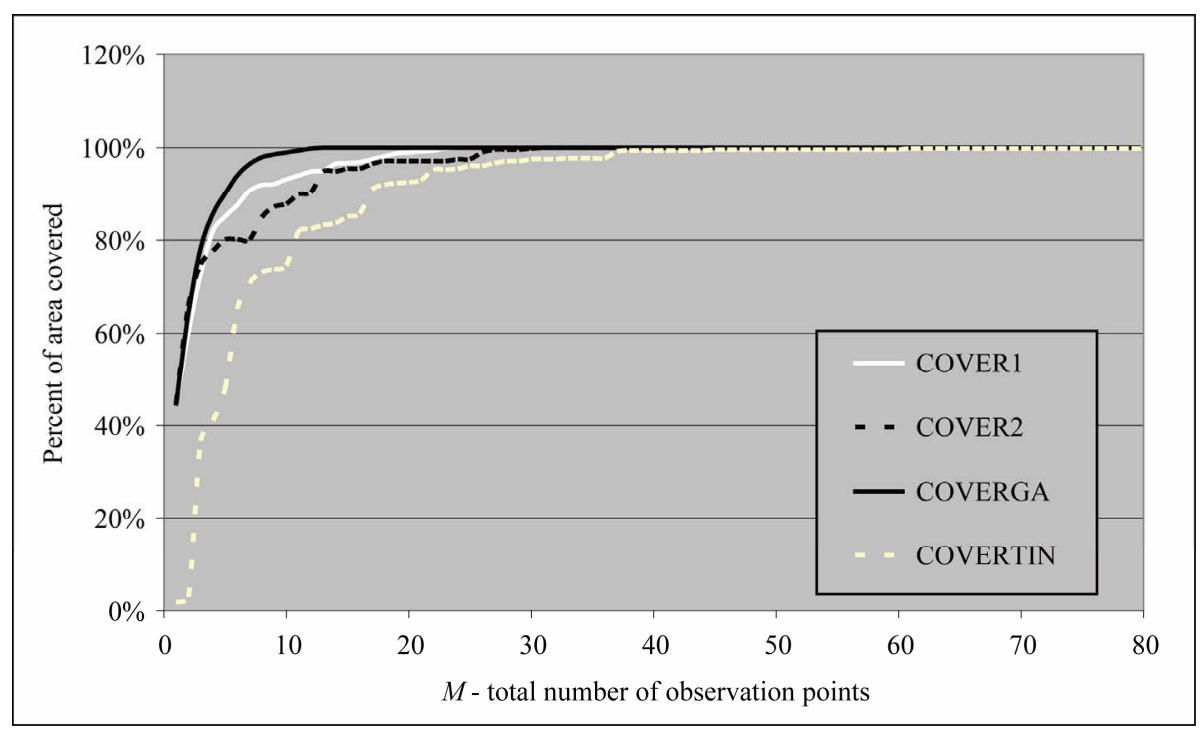

Figure 9. Percentage of area covered by $\mathrm{M}$ observation points.

$3 \mathrm{MHz}$ speed and RAM of 512 megabytes. Sensitivity of the number of observation points to the total number of terrain points covered depicted the classical notion of decreasing returns to scale, increasing in a convex manner as the number of points was increased. Convergence to 100 percent coverage was attained by using $4.9,6.2$, 2.7 and 12.6 percent of the terrain points as observation points for the COVER1, COVER2, COVERGA and COVERTIN, respectively. The best results were for the COVERGA, where by using 13 observation points (only $2.7 \%$ of the points in the terrain) 100 percent coverage was achieved.

\section{Conclusions}

This paper defined a VACS problem and provided two decomposition methodologies: cover first, partition second (CF-PS) and partition first, cover second (PF-CS). Both use heuristic methods to decompose this Np-Hard problem into three main phases. Comparative tests were made for the two methods on real terrains. When comparing the best solutions of both methods the CF-PS method was slightly better. However, because of the increased computation time we suggest that the PF-CS method with the finer TIN terrain resolution be used. This method is faster as partitioning the terrain into separate regions for each observer, provides smaller coverage and routing problems. A sensitivity analysis of the number of observation points to the total number of terrain points covered depicted the classical notion of decreasing returns to scale, increasing in a convex manner as the number of points was increased. The best method achieved 100 percent coverage of the terrain by using only 2.7 percent of its points as observation points.
Several solutions were presented to two military experts, which where responsible for placing observation teams in combat zones. Both experts found the solutions included excellent observation points, with good coverage routes, and that the computerized methodologies were very helpful. They indicated that determining the location of observation points and the routes between them is time consuming when done manually, and until now there had not been a method to establish the quality of a solution. The method can easily be adapted to consider a decreased value of viewing points farther away from the observation point. Also, the method can handle importance weights given to various areas or points in the terrain. The methodology developed can be applied to civilian search operations such as downed planes/lost hikers in remote areas. Also, non dynamic versions of the problem have applications to telecommunication problems such as wireless network coverage.

\section{Acknowledgements}

This research was partially supported by the Paul Ivanier Center for Robotics Research and Production Management, Ben-Gurion University of the Negev.

\section{References}

[1] J. O'Rourke, "Art Gallery Theorems and Algorithms," Oxford University Press, Oxford, 1987.

[2] S. Rana, "Two Approximate Solutions to the Art Gallery Problem," Proceedings of 31st International Conference on Computer Graphics and Interactive Techniques (SIGRAPH), Los Angeles, 8-12 August 2004.

[3] H. Stern, Y. Chassidim and M. Zofi, "Multiagent Visual 
Area Coverage Using a New Genetic Algorithm Selection Scheme," European Journal of Operational Research, Vol. 175, No. 3, 2006, pp. 1890-1907. doi:10.1016/j.ejor.2005.02.078

[4] H. Stern, Y. Chassidim, M. Zofi and M. Kaspi, "Multi Agent Visual Area Coverage Strategies Using an Adaptive Queen Genetic Algorithm," Proceedings of IASTED International Conference on Artificial Intelligence and Applications, Innsbruck, 14-16 February 2005.

[5] W. R. Franklyn, "Applications of Analytical Cartography," Handbook of Discrete and Combinatorial Mathematics, CRC Press, Boca Raton, 2000.

[6] M. Marengoni, B. A. Draper, A. Hanson and R. Sitaraman, "Placing Observers to Cover a Polyhedral Terrain in Polynomial Time," Proceedings of the 3rd IEEE Workshop on Applications of Computer Vision, Sarasota, 2-4 December 1996, pp. 1-6.

[7] V. V. Vazirani, "Approximation Algorithms," SpringerVerlag, Berlin, 2001.

[8] M. R. Garey and D. S. Johnson, "Computers and Intractability: A Guide to the Theory of NP-Completeness," W. H. Freeman and Co, San Francisco, 1979.

[9] H. E. Gindy and D. Avis, "A Linear Algorithm for Computing the Visibility Polygon from a Point," Journal of
Algorithms, Vol. 2, No. 2, 1981, pp. 186-197. doi:10.1016/0196-6774(81)90019-5

[10] D. Cohen-Or and A. Shaked, "Visibility and Dead-Zones in Digital Terrain Maps," Computer Graphics Forum, Vol. 14, No. 3, 1995, pp. 171-180. doi:10.1111/j.1467-8659.1995.cgf143 0171.x

[11] F. M. Jonsson, "An Optimal Pathfinder for Vehicles in Real-World Digital Terrain Maps," The Royal Institute of Science, School of Engineering Physics, Stockholm, 1997.

[12] E. L. Lawler, J. K. Lenstra, A. H. G. R. Kan and D. B. Shmoys, "The Traveling Salesman Problem," John Wiley \& Sons, Hoboken, 1992.

[13] A. Jaszkiewicz, "Comparative Study of Multiple-Objective Metaheuristics on the Bi-Objective Set Covering Problem and the Pareto Memetic Algorithm," Research Report, Institute of Computing Science, Poznan University of Technology, Poznań, 2001.

[14] M. Garland and P. S. Heckbert, "Fast Triangular Approximation of Terrains and Height Fields," Proceedings of SIGGRAPH'97 Conference, Los Angeles, 3-8 August 1997.

[15] B. Hayes, "The Easiest Hard Problem," American Scientist, Vol. 90, No. 2, 2002, pp. 113-117. doi:10.1511/2002.2.113 